\title{
AC 2009-1155: CHANGING THE MARKS BASED CULTURE OF LEARNING THROUGH PEER ASSISTED TUTORIALS
}

Esat Alpay, Imperial College London

Peter Cutler, Imperial College London

Susan Eisenbach, Imperial College London

Anthony Field, Imperial College London 


\title{
Changing the Marks Based Culture of Learning through Peer Assisted Tutorials
}

\author{
E. Alpay ${ }^{1}$, P.S. Cutler ${ }^{2}$, S. Eisenbach ${ }^{2}$ and A.J. Field ${ }^{2}$ \\ 1 Faculty of Engineering (EnVision) \\ 2 Department of Computing \\ Imperial College London, South Kensington Campus, \\ London. SW7 2AZ.
}

Keywords: peer assisted learning; formative assessment; student led tutorials

\begin{abstract}
.
We describe and evaluate an approach to student learning that aims to instil a culture of formative assessment based on peer-assisted self learning, instead of a marks-based culture in which learning effort is rewarded with marks that contribute to the student's degree. The idea is for suitably qualified third- and fourth-year undergraduates to assist in the running of weekly first-year tutorials. They mark submitted work, provide written and verbal feedback on the students' performance and lead problem solving discussions during tutorials. However, contrary to normal practice, the marks they award do not contribute to the students' year total; all tutorial work becomes essentially voluntary. We report results from a pilot implementation of the scheme over a 12-month period in an engineering department at a leading academic institution. The set-up of the scheme was such that a comparative and triangulated assessment was possible amongst the students and tutor team. There was no discernible degradation in student attendance, submission rates and performance in either the weekly exercises or end of year examinations. Further analysis demonstrates that this type of peer-assisted learning improves some key aspects of student learning, and provides important benefits to the senior peers in terms of their own personal development. We conclude that the scheme provides an excellent alternative to traditional learning methods whilst substantially reducing the investment in academic staff time.
\end{abstract}

\section{Introduction.}

Small-group tutorials are widely recognised as one of the most useful forums in first-year university teaching programmes. They provide scope for structured learning through problem solving as well as for informal discussion that helps to instil confidence and to develop a student's critical thinking, organisational and general communication skills. However, successful tutorial support schemes require a great deal of investment in terms of staff time, which is often dominated by tutorial preparation and the marking of weekly exercises. There is thus often a tension between the needs of the academic staff, who need to balance their time between research, administration and other mainstream teaching activities, and that of the students who value enormously the opportunities for learning and feedback that regular small-group tutorials offer. 
As a means of addressing the tension between staff and student needs, peer tutoring provides a method of support in the running of tutorials, whilst potentially reducing the pupil-teacher ratio and allowing timely feedback on each student's progress. Moreover, peer tutor schemes can have widespread learning and development benefits through the facilitation of effective learning communities (Tinto, 2000) and subsequent collaborative pedagogies. Some specific benefits of such peer-based learning have been reported as:

- greater active and student-led involvement with the subject matter (Donelan and Wallace, 1998)

- lower student anxiety and higher student disclosure during tutorial work (Topping, 1998)

- improved subject dialogue within peer groups to support and enhance the feedback process and reflective learning (Nicol and Macfarlane-Dick, 2006), and possibly even overcome liminality when faced with a threshold concept (Meyer and Land, 2005)

- transferable, social and communication skills development (see e.g. Saunders (1992), Topping (1996, 2005), Maheady (1998) and Hirst et al. (2004))

- improved student socialisation and enculturation within the academic environment (Anderson and Boud, 1996)

- improved student perseverance and retention (Tinto, 2000)

Many of the above mentioned benefits are also of relevance to the peer tutors themselves. In addition, the tutoring experience can lead to the development of organisational, communication and critical thinking skills associated with the teaching process, as well as improved knowledge and technical skills through greater attention to and motivation for the subject matter, i.e. learning by teaching (see Hartman (1990) and the discussions of Topping (1996)). Furthermore, the experience may yield motivational and professional-development benefits through greater engagement and identity with academic staff.

Although much research evidence exists in favour of peer tutoring (see e.g. the reviews of Goodlad and Hirst (1989) and Dochy et al. (1999)), the value of such schemes are still challenged by some practitioners. Typical concerns centre around the quality of student tutors and the time and effort needed in the recruitment, training and supervision of the tutors. It has also been argued that whilst a peer tutor may more readily relate to the background knowledge and skills of the student group, the teaching quality may be jeopardised by over emphasis on a strategic approach to learning, rather than any deep and connected understanding of the subject material (Ashwin, 2002). Thus, whilst peer tutoring may help students deal with the demands of the course, it cannot necessarily compensate for a poorly designed course or assessment procedures. Further criticism is also given on the difficulty of establishing effective quantitative measures of impact which can be directly attributed to the peerlearning experience. One approach to address this issue has been the use of student grades and retention/graduation statistics as key measures, with the subsequent 
longitudinal tracking of students who have either experienced or not experienced peer-based supplemental instruction (Congos and Mack, 2005). Yet, with such an approach, questions remain for situations where students are self-selected in learning groups.

In this paper we report our findings from a pilot scheme that employed suitably qualified third- and fourth-year undergraduates (referred to as Undergraduate Teaching Assistants, or UTAs ) to work alongside an academic tutor in the running of weekly first-year tutorials. The UTAs marked the work submitted each week but, unlike the system it replaced, the marks awarded did not count towards the students' final year mark. In this sense the weekly exercises became entirely voluntary. In addition to freeing up a substantial amount of staff time, one of the main objectives was to encourage students to take ownership of their education to a much greater extent than at school, and to motivate the idea that successful learning does not always have to be rewarded by the allocation of marks. We wanted to shift the culture away from a summative, marks-based, type of assessment towards more a formative approach in which (in our case) the act of problem solving in itself is seen as important and rewarding. The sole purpose of the marks issued by the UTAs was thus to provide feedback to the students that they could use to judge their progress and to adjust their own approach to learning.

The structure of the scheme enabled implicit mentoring of peer tutors by academic tutors and, moreover, allowed students within each learning group to comment on the performance of the peer tutor relative to the academic tutor. Thus, whilst the tutorial scheme was designed on the grounds of a student-centred learning experience, a unique opportunity was set-up to evaluate the relative impact of peer-tutoring through groups of students who experience both academic and peer tutors within the same learning context.

The main contribution of the paper is a detailed evaluation of the pilot scheme. The evaluation involves the analysis of data such as student attendance, submission rates and performance in end-of-year examinations, and the results of a series of questionnaires that were completed by the three parties involved in the scheme (students, UTAs and academics). The objectives of the evaluation are to:

- identify the specific areas of benefit offered by considering the views of students, peer tutors and academic tutors (triangulated evaluation)

- illustrate the impact of the scheme on the learning experience relative to academic tutoring (comparative evaluation)

- quantify any significant changes in behaviour or performance, compared with previous years

- identify areas of further development in the set-up and running of the scheme 
Although the evaluation methods were developed for a specific support programme (discrete mathematics for computer science), we anticipate that the design approach will be of value and relevance to other peer-assisted learning schemes.

\section{The Pilot Scheme.}

The Logic, Discrete Mathematics and Reasoning About Programs courses are a core part of the foundation to Computer Science. In the first year of the degree course in question these are taught by lecture courses and supported by weekly small group tutorials (Personal Maths Tutorials, or PMTs) comprising six students and an academic tutor. The group membership is fixed for the year and the tutorials are organized to provide the students with an interactive and practical discussion of a variety of set problems under the guidance of the tutor. An important feature of the PMT system is that work is marked and returned later in the same week that it is completed. Although the tutorial is often used as a workshop for the exercises there is considerable flexibility about the topics discussed. Each week the marks and attendance are recorded on an on-line Continuous Attendance Tracking Engine (CATE) which makes them available to the students and other members of staff. The courses are assessed by a combination of written examinations and continuous assessment, the latter being made up from the marks awarded for the PMT exercises.

The pilot scheme involved reorganizing the weekly PMTs. Each PMT tutor was allocated a student helper (UTA) who took over the running of the tutorials and the marking of the exercises. The role of the academic tutor was to mentor their UTA, to help guide the discussions, and to ensure that the marking of the exercises was consistent and fair. The academics were also responsible for the pastoral care of the students. The weekly exercises were marked and entered into CATE as before, but the marks were zero weighted. The course was thus assessed exclusively by written examinations. To ensure that the UTAs were of high quality, only students with A grades on the degree overall, and in the Mathematics courses being studied, were offered the role. In return, the UTAs were paid on an hourly basis (three hours per week in the pilot scheme).

As part of the pilot scheme we wanted to address the following questions:

- does making the PMT exercises zero weighted have any effect on student motivation and participation in the PMT tutorials?

- how do students react to having tutorials run by students rather than academics?

- do the UTAs find the role rewarding and in what way does it affect their own personal development?

- how do the academics perceive the changes?

- is there a significant difference in student performance as a result of the new scheme? 
The minimum requirement for the modified PMT system to be deemed a success was that it should be at least as effective as the previous scheme in facilitating the student learning of mathematics. In order to evaluate the scheme we used a combination of qualitative and quantitative measures. The former has been obtained from surveys of student participants, student tutors and academics. The latter includes a comparison of student attendance, submission rates and marks achieved in the weekly exercises and final examinations.

\section{Methodology}

The study involved the evaluation of the effectiveness of the use of peer tutors (UTAs) as a supplement to academic tutors in the PMT system. This involved measuring the perceptions of the three different participants: the students, the academics tutors and the UTAs. In order that the results could be triangulated, similar measures were included in the questionnaires that were administered to the different participants. The measures were derived from the learning outcomes expected from the PMT course (Section 3.2).

The student questionnaire was designed to elicit directly a response on the comparative performance of UTAs and academic tutors. This enabled a pairedsamples analysis of the data. The UTAs and academic tutors were given a questionnaire which sought views on the learning and development experiences of the students. The tutor questionnaire also enabled some response on general aspects of the PMT scheme. Data on PMT attendance, assignment submission rate, assignment marks and end-of-year examination results provided a further means of gauging the impact of UTA support, and a quantitative means of assessing the scheme relative to data from previous years.

\subsection{Measures}

The Student Questionnaire

With reference to Appendix 1, the student questionnaire consisted of three sections. Section A contained background information on the participants to allow perceptions of the role of UTAs to be tested against some basic participant data. These include gender, residential status (i.e. home, EU, overseas) and marks achieved on a mid-session test on the course.

Section B of the questionnaire consisted of two parts. The first part (questions 116) was concerned with a direct comparison of the role of UTAs and academic tutors. With reference to Table 1(a), questions were chosen to depict three areas of UTA contribution: motivation and engagement (ME), skills development (SD) and technical explanation, feedback and course contextualisation (EFC). With reference to Table 1(b), the second part of section B (questions 17-30) was concerned with the student connection to the UTA, views on the PMT system (and more generally formative assessment and learning), and student work organisation and time 
management. All the above questions were scored on a 5-point Likert scale, with 1 representing strong disagreement with the statement and 5 strong agreement ${ }^{1}$.

Section $\mathrm{C}$ of the questionnaire consisted of open-ended questions and provided students with an opportunity for further feedback and comment on the PMT system. The questionnaire was piloted on a group of five students to ensure the language was unambiguous and understood by students whose first language was not English. An on-line format for the questionnaire enabled easy student access and data processing.

\section{The UTA / Academic Tutor questionnaire}

With reference to Appendix 2 and Table 2(a), items in the UTA/academic tutor questionnaire were selected to explore perceptions on four themes: the performance and effectiveness of the UTA (UPE), the UTA's personal benefits from the teaching experience (UB), the level of support received by the UTA (US), and student motivations / progress in learning (MP). For the UPE theme, items were selected to explore views relating to the ME, EFC and student connection categories of the student questionnaire. However, it was considered inappropriate to ask a tutor to compare explicitly their performance relative to their academic/student-tutor pair. A 5-point Likert scale was again used for item scoring ${ }^{2}$. Open-ended questions, similar to those in the student questionnaire, also provided the tutors with an opportunity for further elaboration on their experiences.

\subsection{Procedure}

The questionnaires were administered at the end of the course after a complusory on-line test that was attended by all students. Students were informed that the purpose of the questionnaire was to evaluate the use of UTAs. They were also told that the data would be held anonymously. The UTA and academic tutor questionnaire was distributed to the tutors by email, and again anonymity ensured.

The composition of the student group can be summarised as: 112 male (87.5\%); 16 female (12.5); 71 UK home students (55\%); 29 European Union (EU) students (23\%); 28 overseas students $(22 \%)$. The return rate of the student questionnaires was $128 / 132(97 \%)$. For the tutors, a return rate of $17 / 23(74 \%)$ was achieved for both UTA and academic tutors.

\section{Results and Discussion}

\subsection{The student experience}

As mentioned above, for the student questionnaire items 1-16 of part B are divided into three categories: motivation and engagement (ME), skills development (SD), and explanation, contextualisation and feedback (ECF). Principal components

\footnotetext{
1 Responses to items 19, 22, 25 and 26 were transposed such that favourable responses were indicated by a high score.

$2 \quad$ For the purposes of data reduction and scale reliability tests, responses to items 16, 17, 25, 26 and 31 were transposed such that favourable responses were indicated by a high score.
} 
analysis for each of these categories led to single-factor solutions, with corresponding Cronbach alpha coefficients of 0.93, 0.82 and 0.89. The calculations thus confirmed that each set of questions act as a consistent measure of a single underlying dimension.

Descriptive statistics and box-plots of scores for each of the teaching-impact categories are given in Table 3(a) and Figure 1 respectively. The data show that for all categories, the performance of the peer (student) tutors exceeded that of the academic tutors. Nevertheless, scores for both peer and academic tutors were favourable. A paired-samples t-test confirmed the statistical significance of the differences $(\mathrm{p}<0.001)$. With reference to Table 3(b), an item-by-item analysis of questions 1-16 indicated statistically significant differences between scores for the peer and academic tutors (paired samples t-test, $\mathrm{p}<0.05$ ), except for questions 15 and 16 . The three most discriminatory questions (i.e. the questions yielding the maximum difference in mean scores between peer and academic tutors) were indicated as 2, 6, and 8, namely:

"Getting me to participate in the PMT"

"Giving useful feedback on my work"

"Helping me to improve my logic skills"

It is interesting to note that these items represent key facets of the three teachingimpact categories (ME, SD and ECF).

Descriptive statistics for questions 17-30 are given in Table 4; the items are ranked in order of most to least favourable responses. On the basis of the mean overall scores, responses were favourable for all but one question: "I have a fixed timetable to organise my work each week" (question 27). Nevertheless, the positive responses to items 25 and 28 indicate that the students were in fact coping with the time management of their tasks.

Favourable responses to items 17, 18, 20 and 21 can be viewed as representing a positive affinity between the student and the peer tutor. Although a Cronbach alpha calculation indicated a reasonable scale reliability for these 4 items $(\alpha=0.72)$, item 18 ("I would like to be a UTA when I am a final year student") was found to be a marginal component to the scale. Likewise, in retrospection, a negative response to item 21 ("I have a lot in common with the UTA") may not necessarily indicate an unfavourable learning experience, as exemplified by the very high scores for item 17 ("Overall, the UTA has significantly helped me learn the material").

Collective responses to questions $23,24,26,29$ and 30 can be viewed as representing the student attitude towards formative assessment and learning. A principal components analysis indicated that item 26 constituted a separate factor and was thus excluded from this scale. A subsequent Cronbach alpha calculation for items $23,24,29$ and 30 yielded a good scale reliability of 0.75 . The mean value for this scale was calculated as 3.47 , indicating that the students responded well to the PMT teaching initiative. Nevertheless, and not surprisingly, the relatively low (but 
favourable) mean score for item 26 shows that for many students assessed work will take priority.

Analysis based on a gender grouping showed no statistically significant differences in the mean-scores of the 3 teaching-impact categories. Likewise, no differences in scores were found for an item-by-item analysis. Nevertheless, the small sample size of female students should be noted here $(\mathrm{N}=16)$. For the different residential status groupings, again no significant differences were found in the meanscores of the teaching-impact categories. Interestingly though, and item-by-item analysis indicated that EU students generally disagreed more strongly with item 26 ("Non-assessed work is much less important than assessed work") than home or overseas students. No correlation was indicated between exam scores and any of the teaching-impact categories or question items.

Finally, student responses to Section $\mathrm{C}$ showed much appreciation of the PMT scheme and in particular the support given to them by the UTAs, e.g.:

"The UTA scheme was the most helpful learning tool in my course this year."

"It is very helpful as, unlike lectures, the UTA understands what areas we

are unsure of, since he is student after all!"

"Down to earth, jokes, knows the situation that we are in and can relate to it."

"I believe it's helpful, as you can approach the UTA in a more

open manner than you would to with the tutor and that is sometimes helpful."

"He goes through previous exercises very thoroughly and gets each of us to at least attempt to answer questions that the majority of us got incorrect.

"Put a PMT in every course!"

"More sessions per week!! Would improve things hugely!"

"Excellent idea. It both encourages us to aspire to work hard and helps the UTA in furthering his/her skills in teaching."

\subsection{Quantitative measure: attendance, submission rates and marks}

The quantitative measures were provided by comparing attendance, assignment submission rate, marks for PMT submissions and end-of-year examination results for the courses supported by the PMT; see Table 7, which shows the examination mark breakdown by topic. The exam results show fluctuations between the different years related to changes in exam format and the difficulty of the exam set. In particular, the structure of the written examinations was changed in 2007/8 from separate examinations in each of Logic, Program Reasoning and Discrete Mathematics to 'paired' papers in Logic/Reasoning and Continuous/Discrete Mathematics. This could have affected the way students revised for the paper compared with previous years.

The data on the PMT groups show that attendance and submission rates were relatively constant and the marks for the PMT exercises were slightly lower. However, a moderation of a cross-sample of exercise papers gave strong indication that the reduction in the exercise marks were likely to be due to stricter marking by 
the peer tutors. Indeed, the fact that the marks are zero weighted provides some additional freedom to 'mark down' indifferent solutions more heavily than one normally might if the marks were to be counted. With this in mind, the UTAs were encouraged to use the full range of marks available ( $0-10$ for each exercise) to provide rigorous feedback. Although students were not motivated to attend the PMT meeting and complete the exercises by having marks count towards their degree total, they participated fully in the PMT scheme nevertheless.

\subsection{The UTA and academic tutor experience}

For the UTA/academic tutor questionnaire, a principal components analysis on the item list denoted by UPE led to two separate factors:

(i) UPE1: items 3,5,7,9,10,15,19 (alpha $=0.82)$

(ii) UPE2: items 4,11,13,22 (alpha $=0.71)$

UPE1 can be viewed as specific UTA teaching qualities which enhance the student learning experience, such as giving good feedback and explaining material clearly. These items can be seen to relate closely to the EFC category of the student questionnaire. UPE2 can be viewed as some of the affective and social aspects of teaching, such as open communication, better connection to the student's level of understanding, and acting as a tangible role model. These items closely relate to the $\mathrm{ME}$ and student connection categories of the student questionnaire. Items 6, 21, 25 and 30 were treated independently in the data analysis.

For the item list depicted by UB, the exclusion of items 2 and 31 led to a single factor grouping (alpha $=0.73$ ). A direct comparison of items 31 and 32 was undertaken to explore the pay vs. experience motivations of UTAs, and the academic tutor perceptions on this. All five items of the US category led to a single factor grouping (alpha $=0.84$ ). For the MP category, items were treated independently in the data analysis due to poor factorial groupings. A summary of the modified scales after statistical data reduction is given in Table 2(b).

Descriptive statistics for each of the modified scale categories are given in Table 5 for both UTA and academic tutor responses. No statistically significant differences between the UTA and academic tutor scores were found (independent samples t-test), but the low sample size for this data should be noted. Generally, responses on all categories were favourable, with the lowest score corresponding to the views of UTAs on the support they received in running the tutorials.

An item-by-item analysis indicated statistically significant differences in mean scores $(\mathrm{p}<0.05)$ for questions $3,6,20$ and 22 , i.e.:

"The UTA prepares well for the PMT meeting" (Q3)

"The UTA takes the lead role at the PMT meeting" (Q6)

"The UTA and academic discuss teaching strategy before the meeting" (Q20) 
"The UTA is a student so it is easier for him/her to explain concepts to the students" (Q22)

For questions 3 and 6, mean UTA scores were less than academic tutor scores (i.e. 4.06 vs. 4.59 for question 3, and 4.24 vs. 4.88 for question 6). This indicates some underrating of performance by the student tutors, or indeed overrating of UTA performance by academic tutors of the UTA performance. Nevertheless, the responses were favourable by both sets of tutors. For question 20, differing views were indicated, with the UTA mean score (2.45) showing slight disagreement with the statement and the academic tutor mean score (3.47) showing agreement. For question 22 , in general UTAs were in agreement with this statement (mean score $=3.88$ ), whereas academic tutors were neutral (mean score $=2.94$ ).

With reference to Table 6, mean item scores showed that generally both UTAs and academic tutors were in disagreement with questions 16, 26 and 31, i.e. mean scores < 3. In other words, favourable responses were given to the negatives items of:

"Most students are concerned with marks than understanding" (Q16)

"The academic tutor finds it hard to get the tutor to participate" (Q26)

"The payment is the most important motivator for a UTA" (Q31)

However, a clear disagreement in views were found for item 20 ("The UTA and academic tutor discuss teaching strategy before the meeting"), with UTAs generally disagreeing with this statement $(\mathrm{p}<0.01)$. Nevertheless, UTAs and academic tutors indicated mean score ratings $>4$ (i.e. agreement) for approximately half the items, with 12 common items amongst these. Of these 12 items, 8 corresponded to UTA performance and effectiveness (UPE). It is interesting to note that academic tutors felt most positive about the skills development of UTAs themselves, and UTA preparation and initiative in running the tutorials. The UTAs also rated highly the skills development benefits which they have gained as tutors. In terms of the gross motivations of UTAs, experience rather than payment was clearly indicated.

Finally, comments by the academic tutors showed that they valued the scheme and in particular were happy not to be marking the exercises.

"[the scheme has led to] More flexibility - lighter work load"

"UTA develops useful skills and acquires confidence. He/she feels part of the department"

"They do the marking! Also it is interesting to see how someone else tries to explain the technical things"

Comments by UTAs were also favourable, e.g.:

"Experience gained - teaching, assessing. Seeing students develop/overcome learning barriers." 
"The meetings are fun and it is good talking with the students and getting them involved in the subject"

However, some of the academic tutors and UTAs felt that there was an issue with the authority of the UTA especially when they were both present at the tutorial. One UTA noted:

"I don't think the [academic] should be present; this undermines the UTA's authority"

Whilst one of the academics expressed the view that:

"UTAs lack some authority: the [academic] should attend the meetings."

Concerns about the UTA's authority were not apparent from the student comments; generally they wanted more PMT sessions.

\section{Further Discussion}

The above results show that the modifications to the PMT system was positively evaluated by the students, UTAs and academic tutors. The students rated the UTAs more highly than the academic tutors in areas concerning motivating and engaging teaching, skills development facilitation, and the quality of technical explanations, feedback and course contextualisation. There was no decrease in assignment submission rates compared to previous years despite the work being zero weighted. It should be noted however that the presence of academic tutors in peer-led tutorials, as well as their mentoring role towards the peer tutors, is likely to have influenced the favourable performance of the peer tutors. It is encouraging, though, that items 2, 6 and 8 of the student questionnaire were particularly discriminating between peer and academic tutor evaluations. As discussed earlier, some of the key benefits of peer learning schemes are to promote wider student participation, improve feedback and encourage greater engagement with the subject matter. The student responses to items 2, 6 and 8 suggest that indeed such outcomes are being achieved. Enthusiasm for the UTAs was also reflected in the student answers to open-ended questions, and further demonstrate that the PMT scheme worked well to increase student motivation and discourage the adoption of a marks based culture.

Although the data suggests favourable student attitudes towards formative assessment, inevitably student priority and concerns remain towards assessed work. An advantage of the PMT scheme is its close integration with the course, with structured assessment exercises which complement course outcomes, and ultimately the final exam. Here again, the point that peer tutoring by itself cannot compensate for poor course design and assessment procedures is clear (Ashwin, 2002). Student comments which refer to the scheme as a learning tool for the course clearly exemplify this complementary design. 
The UTAs also found the PMT experience valuable, with intrinsic motivations towards the teaching experience itself. The academics particularly appreciated the gain in time from not having to mark the exercises. Interestingly, although academic ratings of the UTA teaching quality featured high, there is a persistent notion by some academics that peer tutors do not necessarily have an advantage in explaining concepts to the student group. In the PMT scheme, however, this belief is not in accordance with the student responses. A further positive outcome of the UTA/academic tutor questionnaire was that some of the specific support needs of the peer tutors were identified. These included guidance on marking schemes and on teaching strategies prior to a tutorial.

In the design of the student questionnaire, it was helpful to select items which corresponded to pertinent categories of the learning experience. The statistical grouping of question items led to further assurance of a broader impact on learning. However, further scope exists in the questionnaire design to better explore specific transferable, study and communication skills. Nevertheless, as a means of capturing the relative performance of peer tutors in concise way, the set-up of direct comparative questions between academic and peer tutors was found to be particularly effective, and alleviated issues associated with any subjective interpretation of the questions, and indeed psychological influences such as the Hawthorne effect (see e.g. Draper, 2005).

Having established clearer question groupings (scales) for the student questionnaire, in future work more direct mapping between the student and tutor questionnaires would be advantageous. Such an approach would lead to greater triangulation in the interpretation of the student experience. However, as a means of evaluating peer tutoring within the pilot study, the exercise has demonstrated the advantages of multiple assessments which involve, for example, the achievement and completion type indicators proposed by Congos and Mack (2005), as well as attitudinal and descriptive information from both students and tutors.

\section{Conclusions}

The scheme supports the view that fostering a marks based culture does not motivate students to learn. Rather it is possible to move towards encouraging students to learn based on the intrinsic value of the material. The combined use of student tutors (UTAs) and an academic tutor in the running of tutorial sessions proved extremely successful and was popular with the students. The UTA scheme allowed academics to focus on the pastoral aspects of teaching and encouraged the students to take ownership of their learning. The UTAs themselves additionally felt that they had developed their own communicative skills as a result of the scheme. Making the exercises zero weighted had no significant negative impact on student participation in the tutorials or examination results. A very significant result was that the students saw the UTAs as role models since they were students like them, but who had already successfully achieved mastery in the subject. Hence, both making the exercises zero 
weighted and using student tutors was successful in changing quite fundamentally the learning culture in the department.

\section{Acknowledgements}

The authors would like to thank Duncan White of CSG for developing the online questionnaire software Q. 


\section{References.}

Anderson, G., Boud, D., 1996, Extending the role of peer learning in university courses. Research and Development in Higher Education, 19, 15-19

Congos, D.H., Mack, A., 2005, Supplemental instruction's impact in two freshman chemistry classes: research, modes of operation, and anecdotes. Research \& Teaching in Developmental Education, 21 (2), p. 43-64.

Dochy, F., Segers, M., Sluijsmans, 1999, The use of self-, peer and co-assessment in higher education: a review. D, Studies in Higher Education, 24 (3), p. 331-350

Donelan, M., Wallace, J., 1998, Peer support programmes - a truly co-operative initiative. In Dolan, J., Castley, A.J.: Students supporting students. SEDA Paper 105, Birmingham, Staff and Educational Development Association, p. 11-22.

Draper, S.W., 2005, The Hawthorne, Pygmalion, placebo and other expectancy effects: some notes. URL: http://www.psy.gla.ac.uk/ steve/hawth.html (visited February 2009)

Goodlad, S., Hirst, B., 1989, Peer Tutoring: A Guide to Learning by Teaching, London: Kogan

Hartman, H.J., 1990), Factors affecting the tutoring process. Journal of Educational Development, $14(2), 2-6$.

Maheady, L.,1998, Advantages and disadvantages of peer-assisted learning strategies. In: Peer-assisted learning, Topping, K. (Ed.) (pp. 45-62). Mahwah, NJ: Lawrence Erlbaum Associates.

Meyer, J.H.F., Land, R., 2005, Threshold concepts and troublesome knowledge (2): Epistemological considerations and a conceptual framework for teaching and learning. Higher Education, 49. p. $373-$ 388

Nicol, D.J., Macfarlane-Dick, D., 2006, Formative assessment and self-regulated learning: a model and seven principles of good feedback practice. Studies in Higher Education, 31(2), p. 199-218

Saunders, D., 1992, Peer tutoring in higher education. Studies in Higher Education, 17(2), p. 211-218

Tinto, V., 2000, Learning better together: the impact of learning communities on student success in Higher Education, Journal of Institutional Research, 9(1), 48-53

Topping, K.J., 1996, The effectiveness of peer tutoring in further and higher education: a typology and review of the literature. Higher Education ,32. p. 321-345

Topping, K.J., 1998, The Effectiveness of peer tutoring in further and higher education: a typology and review of the literature". In: Goodlad, S. (ed), Mentoring and Tutoring by Students, London: Kogan, p. 49-69

Topping, K.J., 2005, Trends in peer learning. Educational Psychology, 25 (6), p. 631-645 


\section{Appendix 1 - The Student Questionnaire (condensed format).}

We would like your views on the use of final year students to help with the small group tutorials associated with the PMT work, so could you please complete the questionnaire below. The survey will not take long to complete.

Note: $P M T=$ Personal Maths Tutor or Personal Maths Tutorial depending on context and UTA = Undergraduate Teaching Associate.

All responses will be kept confidential and will only be used for the purposes of statistical analysis.

Key for responses:

1 =strongly agree; 2 =agree 3 = neither agree nor disagree $4=$ disagree $5=$ strongly disagree

\section{A: Background Information}

\begin{tabular}{|l|l|l|l|l|l|l|l|l|l|}
\hline 1) Gender & \multicolumn{2}{l|}{ Male } & Female \\
\hline 2) UTA Gender & \multicolumn{2}{l|}{ Male } & Female \\
\hline 3) PMT Gender & \multicolumn{2}{l|}{ Male } & Female \\
\hline 4) Residential Status & \multicolumn{2}{l|}{ Home (UK) } & \multicolumn{2}{|l|}{ EU } & Overseas \\
\hline 5) Grade in the Xmas Test & A & A & B & C & D & E & F & Can't remember \\
\hline
\end{tabular}

B(1): Evaluation of PMT and UTA

\begin{tabular}{|c|c|c|c|c|c|c|c|c|c|c|}
\hline \multirow[b]{2}{*}{ 1) Understanding my needs as a first year student } & \multicolumn{5}{|c|}{ UTA } & \multicolumn{5}{|c|}{ PMT } \\
\hline & 1 & 2 & 3 & 4 & 5 & 1 & 2 & 3 & 4 & 5 \\
\hline 2) Getting me to participate in the PMT & 1 & 2 & 3 & 4 & 5 & 1 & 2 & 3 & 4 & 5 \\
\hline 3) Making the PMT work interesting & 1 & 2 & 3 & 4 & 5 & 1 & 2 & 3 & 4 & 5 \\
\hline 4) Making it easy for me to talk about things I don't understand & 1 & 2 & 3 & 4 & 5 & 1 & 2 & 3 & 4 & 5 \\
\hline 5) Having a difficult concept explained to me & 1 & 2 & 3 & 4 & 5 & 1 & 2 & 3 & 4 & 5 \\
\hline 6) Giving useful feedback on my work & 1 & 2 & 3 & 4 & 5 & 1 & 2 & 3 & 4 & 5 \\
\hline 7) Clarifying/correcting misunderstanding & 1 & 2 & 3 & 4 & 5 & 1 & 2 & 3 & 4 & 5 \\
\hline 8) Helping me improve my logic skills & 1 & 2 & 3 & 4 & 5 & 1 & 2 & 3 & 4 & 5 \\
\hline 9) Helping me improve my communication skills & 1 & 2 & 3 & 4 & 5 & 1 & 2 & 3 & 4 & 5 \\
\hline 10) Exploring a problem/concern thoroughly & 1 & 2 & 3 & 4 & 5 & 1 & 2 & 3 & 4 & 5 \\
\hline 11) Motivating me to want to do well on the course & 1 & 2 & 3 & 4 & 5 & 1 & 2 & 3 & 4 & 5 \\
\hline 12) Giving me reassurance about my learning progress & 1 & 2 & 3 & 4 & 5 & 1 & 2 & 3 & 4 & 5 \\
\hline 13) Clarifying why the material will be useful later on & 1 & 2 & 3 & 4 & 5 & 1 & 2 & 3 & 4 & 5 \\
\hline 14) Helping me to manage my time so that I can finish the exercises & 1 & 2 & 3 & 4 & 5 & 1 & 2 & 3 & 4 & 5 \\
\hline 15) Making me feel part of the department & 1 & 2 & 3 & 4 & 5 & 1 & 2 & 3 & 4 & 5 \\
\hline 16) Exploring related topics not covered directly by the course & 1 & 2 & 3 & 4 & 5 & 1 & 2 & 3 & 4 & 5 \\
\hline
\end{tabular}


B(2): General Questions

\begin{tabular}{|c|c|c|c|c|c|}
\hline 17) Overall the UTA has significantly helped me learn the material & 1 & 2 & 3 & 4 & 5 \\
\hline 18) I would like to be a UTA when I am a final year student & 1 & 2 & 3 & 4 & 5 \\
\hline 19) I get more help from my friends than the PMT session & 1 & 2 & 3 & 4 & 5 \\
\hline 20) I am able to approach the UTA outside the PMT meeting & 1 & 2 & 3 & 4 & 5 \\
\hline 21) I have a lot in common with the UTA & 1 & 2 & 3 & 4 & 5 \\
\hline 22) I don't like to admit that I don't know something at the PMT meeting & 1 & 2 & 3 & 4 & 5 \\
\hline 23) I find solving a difficult PMT question gives me satisfaction & 1 & 2 & 3 & 4 & 5 \\
\hline 24) I think that the PMT work is just as important as the practical coursework & 1 & 2 & 3 & 4 & 5 \\
\hline 25) I have difficulty with managing my time to do all the coursework set & 1 & 2 & 3 & 4 & 5 \\
\hline 26) Non-assessed work is much less important than assessed work & 1 & 2 & 3 & 4 & 5 \\
\hline 27) I have a fixed timetable to organise my work each week & 1 & 2 & 3 & 4 & 5 \\
\hline 28) I plan my work with reference to CATE & 1 & 2 & 3 & 4 & 5 \\
\hline 29) I complete all the PMT questions set each week & 1 & 2 & 3 & 4 & 5 \\
\hline 30) Overall I find the PMT work useful and interesting & 1 & 2 & 3 & 4 & 5 \\
\hline
\end{tabular}

C: Open Ended Questions

31) Do you have any comments on the way the UTA scheme is working for the PMT course?

32) What do you like most about the way the UTA runs the PMT meeting/gives you feedback on your work?

33) What do you like least about the way the UTA runs the PMT meeting/gives you feedback on your work?

34) What improvements do you think can be made on the PMT system? 


\section{Appendix 2 - UTA / Academic Tutor Questionnaire (condensed format)}

The survey will not take very long to complete and will be used for evaluating the use of UTAs for the PMT system.

All responses will be kept confidential and will only be used for the purposes of statistical analysis.

Note for academics (PMTs) - could you please answer the questions with reference to your personal experience of working with the UTA assigned to help you.

Note for UTAs - could you please answer the questions with reference to your personal experience of working with the academic (PMT) you have been assigned to help.

Note: $P M T=$ Personal Maths Tutor or Personal Maths Tutorial depending on context and UTA = Undergraduate Teaching Associate.

Key for responses: 1 =strongly agree; 2 =agree 3 = neither agree nor disagree $4=$ disagree $5=$ strongly disagree

A: Evaluation of PMT

1) The UTA gets good support for his/her role

2) The UTA's communication skills are improving over time

3) The UTA prepares well for the PMT meeting

4) The UTA is a role model for the first year students

5) The UTA has many interests in common with the students

6) The UTA takes the lead role at the PMT meeting

7) The UTA uses initiative in running the PMT meeting

8) The UTA gets good support from the academic (PMT)

9) The UTA gives thorough feedback to the students

10) The UTA explains the PMT material clearly

11) The students find it easy to talk openly to a UTA

12) The students find it easy to talk freely with the academic (PMT)

13) The students want the UTA to think well of them

14) The students want the academic (PMT) to think well of them

15) The students find it easy to ask a UTA when they don't understand

16) Most students are more concerned with marks than understanding

17) I suspect that some of my students copy their PMT submissions

18) The students take the feedback on their work seriously

19) The UTA has a clear idea about how to run the PMT meeting

20) The UTA and academic (PMT) discuss teaching strategy before the meeting

21) I enjoy seeing the students develop their skills

22) The UTA is a student so it easier for him/her to explain concepts to the PMT students

23) The students have improved in the PMT meetings over the year

24) The mentoring role of academic (PMT) is very important for a UTA

25) The UTA finds it hard to get the students to participate

26) The academic (PMT) finds it hard to get the students to participate

27) The UTA finds it helpful to revisit the first year material

28) The academic (PMT) gives the UTA useful advice about running the PMT

29) The UTA learns useful transferable skills by helping with the PMT

30) Having a UTA helps enhance the department's intellectual culture

31) The payment is the most important motivator for a UTA

32) The experience is the most important motivator for a UTA

\begin{tabular}{|l|l|l|l|l|}
\hline 1 & 2 & 3 & 4 & 5 \\
\hline 1 & 2 & 3 & 4 & 5 \\
\hline 1 & 2 & 3 & 4 & 5 \\
\hline 1 & 2 & 3 & 4 & 5 \\
\hline 1 & 2 & 3 & 4 & 5 \\
\hline 1 & 2 & 3 & 4 & 5 \\
\hline 1 & 2 & 3 & 4 & 5 \\
\hline 1 & 2 & 3 & 4 & 5 \\
\hline 1 & 2 & 3 & 4 & 5 \\
\hline 1 & 2 & 3 & 4 & 5 \\
\hline 1 & 2 & 3 & 4 & 5 \\
\hline 1 & 2 & 3 & 4 & 5 \\
\hline 1 & 2 & 3 & 4 & 5 \\
\hline 1 & 2 & 3 & 4 & 5 \\
\hline 1 & 2 & 3 & 4 & 5 \\
\hline 1 & 2 & 3 & 4 & 5 \\
\hline 1 & 2 & 3 & 4 & 5 \\
\hline 1 & 2 & 3 & 4 & 5 \\
\hline 1 & 2 & 3 & 4 & 5 \\
\hline 1 & 2 & 3 & 4 & 5 \\
\hline 1 & 2 & 3 & 4 & 5 \\
\hline 1 & 2 & 3 & 4 & 5 \\
\hline 1 & 2 & 3 & 4 & 5 \\
\hline 1 & 2 & 3 & 4 & 5 \\
\hline 1 & 2 & 3 & 4 & 5 \\
\hline 1 & 2 & 3 & 4 & 5 \\
\hline 1 & 2 & 3 & 4 & 5 \\
\hline 1 & 2 & 3 & 4 & 5 \\
\hline 1 & 2 & 3 & 4 & 5 \\
\hline 1 & 2 & 3 & 4 & 5 \\
\hline 1 & 2 & 3 & 4 & 5 \\
\hline 1 & 2 & 3 & 4 & 5 \\
\hline & & & \\
\hline
\end{tabular}




\section{UTA / Academic Tutor Questionnaire (continued)}

B: Open Ended Questions

33 Do you have any comments on the way the UTA program is working?

34 What do you like most about having a UTA?

35 What do you like least about having a UTA?

36 What works well with the UTA program?

37 What doesn't work well with the UTA program?

38 What improvements do you think can be made to the UTA program?

39 Any further comments? 
$\underline{\text { Figures and Tables }}$

Figure 1 - Box-plots of student responses for the teaching categories of motivation and engagement (ME), skills development (SD), and explanation, contextualisation and feedback (ECF). Data is shown for views towards both peer and academic tutors.

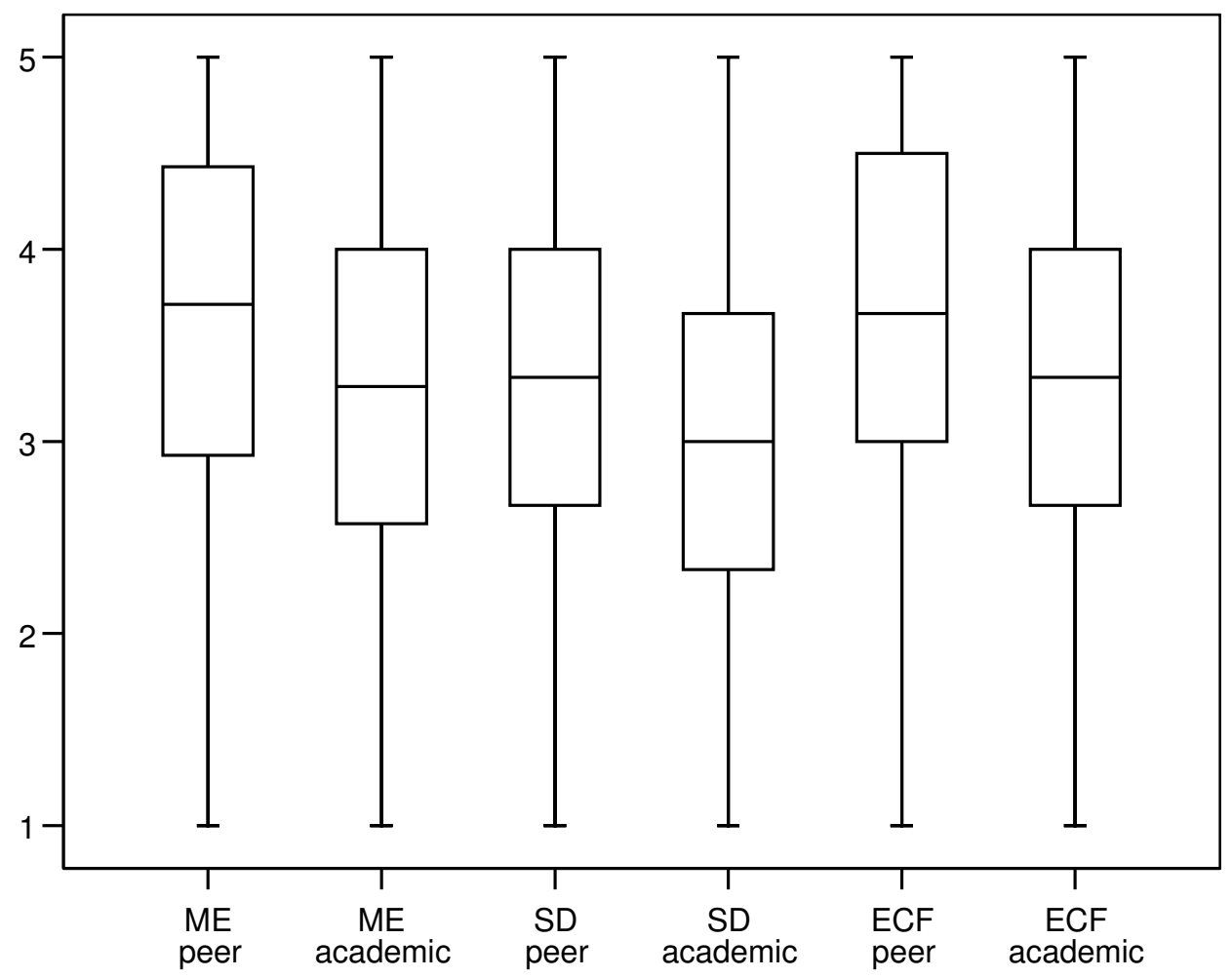


Table 1 - Item categorisation for the student questionnaire for (a) part B, section 1, and (b) part B, section 2. Items in brackets indicate their subsequent removal in the data analysis of a unified category scale.

\begin{tabular}{|ll|l|}
\hline category & item \\
\hline (a) & mE $\quad$ skills development & $1,2,3,4,11,12,15$ \\
\hline SD $\quad \begin{array}{l}\text { explanation, feedback and course } \\
\text { contextualisation }\end{array}$ & $5,6,7,10,13,16$ \\
\hline EFC & student connection with the UTA & $17,18,20,21$ \\
\hline $\begin{array}{l}\text { views on PMT system / formative } \\
\text { assessment and learning }\end{array}$ & $19,22,23,24,(26), 29,30$ \\
\hline $\begin{array}{l}\text { work organisation and time } \\
\text { management } \\
\text { (items treated independently) }\end{array}$ & $(25),(27),(28)$ \\
\hline
\end{tabular}

Table 2 - Item categorisation for the UTA / academic tutor questionnaire: (a) original categorisation, (b) categorisation following statistical data reduction.

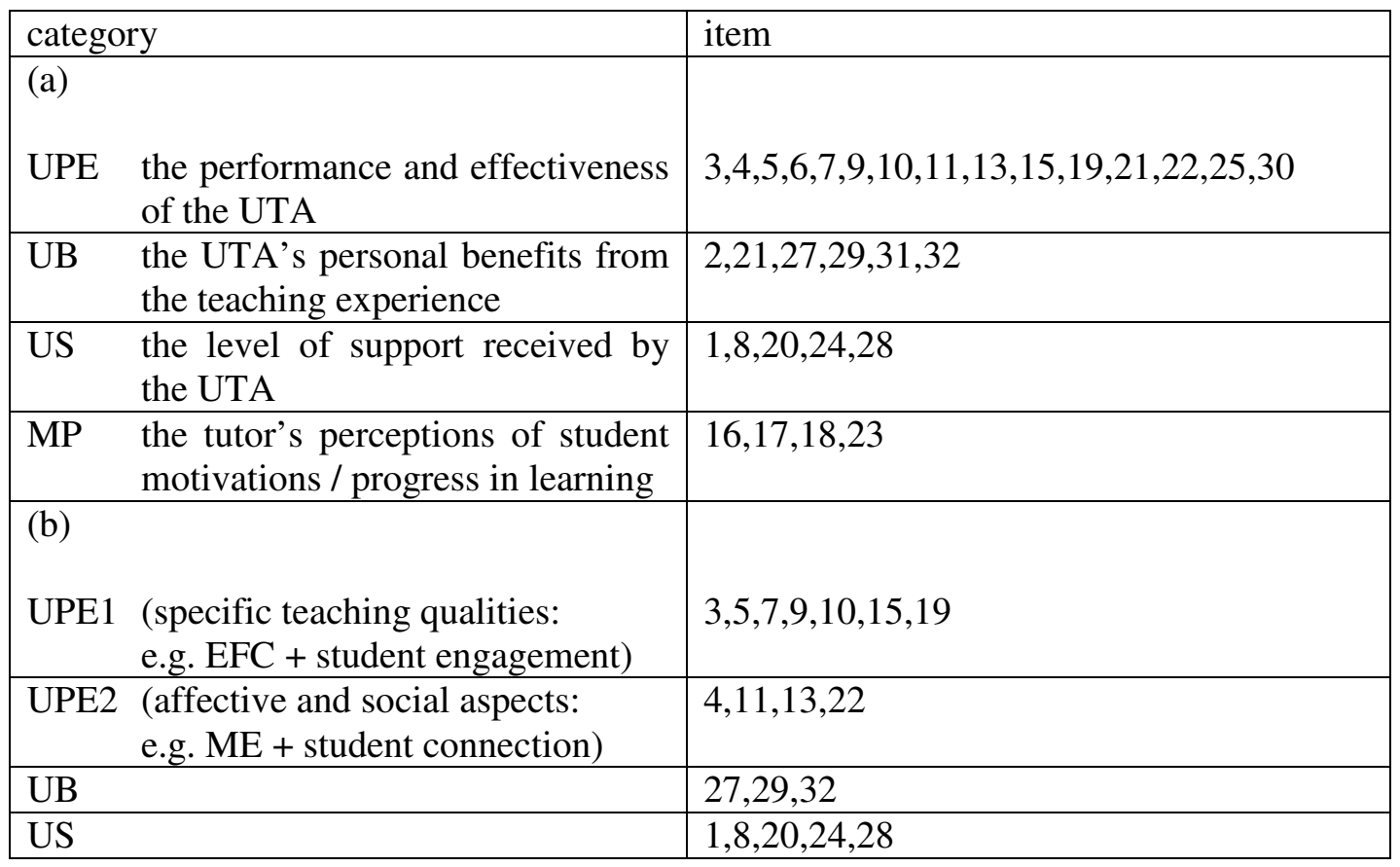


Table 3 - (a) Descriptive statistics for the 3 teaching-impact categories: ME, SD and ECF. (b) An item-by-item analysis of questions 1-16 showing mean score differences between the student ratings of UTAs and academic tutors; statistical significance measures shown using a paired samples t-test.

(a)

\begin{tabular}{|l|l|l|}
\hline & mean & $\begin{array}{l}\text { standard } \\
\text { deviation }\end{array}$ \\
\hline ME peer & 3.60 & 0.99 \\
ME academic & 3.20 & 1.10 \\
SD peer & 3.38 & 0.99 \\
SD academic & 3.01 & 1.06 \\
ECF peer & 3.63 & 0.97 \\
ECF academic & 3.19 & 1.03 \\
\hline
\end{tabular}

(b)

\begin{tabular}{|l|l|l|l|l|}
\hline & $\begin{array}{l}\text { mean } \\
\text { difference }\end{array}$ & $\begin{array}{l}\text { standard } \\
\text { deviation }\end{array}$ & $\mathrm{t}$ & $\mathrm{p}$ (2-tailed) \\
\hline B01 & -0.49 & 1.37 & -4.05 & 0.000 \\
B02 & -0.57 & 1.58 & -4.09 & 0.000 \\
B03 & -0.40 & 1.67 & -2.70 & 0.008 \\
B04 & -0.45 & 1.55 & -3.25 & 0.001 \\
B05 & -0.38 & 1.57 & -2.75 & 0.007 \\
B06 & -0.72 & 1.50 & -5.44 & 0.000 \\
B07 & -0.53 & 1.46 & -4.11 & 0.000 \\
B08 & -0.58 & 1.46 & -4.49 & 0.000 \\
B09 & -0.27 & 1.38 & -2.18 & 0.031 \\
B10 & -0.44 & 1.54 & -3.22 & 0.002 \\
B11 & -0.36 & 1.45 & -2.81 & 0.006 \\
B12 & -0.37 & 1.46 & -2.84 & 0.005 \\
B13 & -0.46 & 1.40 & -3.72 & 0.000 \\
B14 & -0.27 & 1.25 & -2.48 & 0.014 \\
B15 & -0.21 & 1.21 & -1.97 & 0.052 \\
B16 & -0.10 & 1.38 & -0.83 & 0.406 \\
\hline
\end{tabular}


Table 4 - Descriptive statistics for questions 17-30 ranked in order of most to least favourable responses.

\begin{tabular}{|l|l|l|}
\hline & mean & $\begin{array}{l}\text { standard } \\
\text { deviation }\end{array}$ \\
\hline B17 & 3.73 & 1.23 \\
B23 & 3.59 & 1.18 \\
B30 & 3.59 & 1.11 \\
B28 & 3.58 & 1.27 \\
B24 & 3.55 & 1.21 \\
B22 & 3.41 & 1.28 \\
B18 & 3.21 & 1.34 \\
B19 & 3.20 & 1.30 \\
B20 & 3.19 & 1.34 \\
B29 & 3.13 & 1.29 \\
B25 & 3.13 & 1.18 \\
B26 & 3.01 & 1.23 \\
B21 & 2.82 & 1.15 \\
B27 & 2.19 & 1.14 \\
\hline
\end{tabular}

Table 5 - Descriptive statistics for each of the modified scale categories given in Table 2(b).

\begin{tabular}{|c|c|c|c|}
\hline & & mean & $\begin{array}{l}\text { standard } \\
\text { deviation }\end{array}$ \\
\hline \multirow{2}{*}{ UPE1 } & UTA & 4.02 & 0.45 \\
\hline & PMT & 4.34 & 0.56 \\
\hline \multirow[t]{2}{*}{ UPE2 } & UTA & 3.85 & 0.69 \\
\hline & PMT & 3.67 & 0.80 \\
\hline \multirow[t]{2}{*}{ UB } & UTA & 4.08 & 0.64 \\
\hline & PMT & 3.90 & 0.57 \\
\hline \multirow[t]{2}{*}{ US } & UTA & 3.42 & 0.72 \\
\hline & PMT & 3.86 & 0.79 \\
\hline
\end{tabular}


Table 6 - Descriptive statistics for all questions in the UTA / academic tutor questionnaire. Questions are ranked in order of descending UTA mean scores. Note: (i) Shaded items indicate scores $>4$ common to both UTA and academic tutor responses, (ii); items in bold show scores $<3$ common to both UTA and academic tutor responses; (iii) * denotes items in which a low score represents a favourable response; (iv) question categories are indicated.

\begin{tabular}{|c|c|c|c|c|c|}
\hline & \multicolumn{2}{|c|}{ UTA } & \multicolumn{2}{|c|}{ academic tutor } \\
\hline & & mean & $\begin{array}{l}\text { standard } \\
\text { deviation }\end{array}$ & mean & $\begin{array}{l}\text { standard } \\
\text { deviation }\end{array}$ \\
\hline Q21 & (UB) & 4.53 & .80 & 4.38 & 1.26 \\
\hline Q29 & UB & 4.47 & .72 & 4.56 & 0.73 \\
\hline Q23 & (MP) & 4.41 & .62 & 4.06 & 0.77 \\
\hline Q2 & (UB) & 4.41 & .62 & 3.88 & 1.31 \\
\hline Q32 & UB & 4.29 & .69 & 3.88 & 0.89 \\
\hline Q6 & (UPE) & 4.24 & .75 & 4.94 & 0.25 \\
\hline Q11 & UPE2 & 4.18 & .81 & 4.13 & 1.36 \\
\hline Q9 & UPE1 & 4.18 & .53 & 4.44 & 0.63 \\
\hline Q7 & UPE1 & 4.18 & .73 & 4.50 & 0.63 \\
\hline Q19 & UPE1 & 4.12 & .33 & 4.38 & 0.89 \\
\hline Q1 & US & 4.12 & .86 & 4.13 & 1.26 \\
\hline Q30 & (UPE) & 4.06 & .83 & 4.38 & 0.81 \\
\hline Q10 & UPE1 & 4.06 & .56 & 4.50 & 0.73 \\
\hline Q3 & UPE1 & 4.06 & .56 & 4.63 & 0.62 \\
\hline Q15 & UPE1 & 3.94 & .90 & 4.38 & 0.50 \\
\hline Q8 & US & 3.94 & .83 & 3.81 & 1.22 \\
\hline Q22 & UPE2 & 3.88 & 1.27 & 3.00 & 1.16 \\
\hline Q4 & UPE2 & 3.76 & .83 & 4.38 & 0.81 \\
\hline Q18 & (MP) & 3.76 & .44 & 4.13 & 0.72 \\
\hline Q14 & & 3.71 & .69 & 3.50 & 1.10 \\
\hline Q13 & UPE2 & 3.59 & .62 & 3.19 & 1.05 \\
\hline Q5 & UPE1 & 3.59 & .87 & 3.56 & 1.26 \\
\hline Q24 & US & 3.47 & .94 & 3.88 & 0.89 \\
\hline Q27 & UB & 3.47 & 1.07 & 3.25 & 1.24 \\
\hline Q12 & (UPE) & 3.29 & 1.16 & 3.25 & 1.44 \\
\hline Q17* & $(\mathrm{MP})$ & 3.24 & 1.44 & 2.63 & 1.36 \\
\hline Q28 & US & 3.12 & 1.05 & 3.81 & 1.22 \\
\hline Q26* & & 2.53 & 1.28 & 2.56 & 1.41 \\
\hline Q25* & (UPE) & 2.53 & 1.37 & 3.00 & 1.46 \\
\hline Q20 & US & 2.47 & 1.12 & 3.69 & 0.95 \\
\hline Q16* & (MP) & 2.41 & 1.12 & 2.50 & 0.73 \\
\hline Q31* & (UB) & 2.41 & .94 & 2.63 & 0.81 \\
\hline
\end{tabular}


Table 7 - Quantitative Measures of attendance, submission rate, marks achieved and examination results.

\begin{tabular}{|l|c|c|c|}
\hline & $\begin{array}{c}2005-2006 \\
(\%)\end{array}$ & $\begin{array}{c}2006-2007 \\
(\%)\end{array}$ & $\begin{array}{c}2007-2008 \\
(\%)\end{array}$ \\
\hline student attendance at PMT sessions & 89.2 & 92.3 & 92.7 \\
\hline submission rate (PMT work) & 93.5 & 98.3 & 96.8 \\
\hline average mark (PMT work) & 80.0 & 81.4 & 71.2 \\
\hline test result (Logic + Discrete Maths) & 48.9 & 53.0 & 57.0 \\
\hline Discrete Mathematics exam average & 64.3 & 59.8 & 57.1 \\
\hline Logic exam average & 55.0 & 61.7 & 60.4 \\
Reasoning About Programs exam average & 53.7 & 55.1 & 53.8 \\
\hline
\end{tabular}

